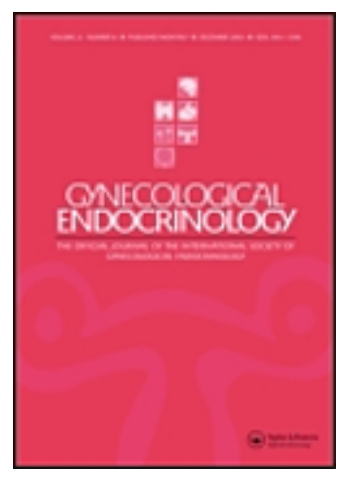

\title{
Clinical Performance of a specific Granulocyte Colony Stimulating Factor ELISA to Determine its Concentration in Follicular Fluid as a Predictor of Implantation Success during In Vitro Fertilization
}

\begin{tabular}{|c|c|}
\hline Journal: & Gynecological Endocrinology \\
\hline Manuscript ID & DGYE-2019-0269 \\
\hline Manuscript Type: & Original Paper \\
\hline $\begin{array}{r}\text { Date Submitted by the } \\
\text { Author: }\end{array}$ & 08-May-2019 \\
\hline Complete List of Authors: & $\begin{array}{l}\text { Tournaye, Herman; UZ Brussel, } \\
\text { D'Hooghe, Thomas; KU Leuven University Hospitals Leuven, Department } \\
\text { of Development and Regeneration } \\
\text { Verheyen, Greta; UZ Brussel } \\
\text { Devreker, Fabienne; Université Libre de Bruxelles Faculté de Médecine } \\
\text { PERRIER d'HAUTERIVE, Sophie; CPMA - University of Liège, CHR } \\
\text { Citadelle - Gynaecology and Obstetrics } \\
\text { NISOLLE, Michelle; CPMA - University of Liège, CHR Citadelle - } \\
\text { Gynaecology and Obstetrics } \\
\text { Foidart, Jean-Michel; University of Liege, Laboratory of Tumor and } \\
\text { Development Biology } \\
\text { Munaut, Carine; University of Liege, Laboratory of Tumor and } \\
\text { Development Biology } \\
\text { Noël, Laure; University of Liege, Laboratory of Tumor and Development } \\
\text { Biology; Liege University, Centre de Procréation Médicalement Assistée }\end{array}$ \\
\hline Keywords: & $\begin{array}{l}\text { granulocyte colony stimulating factor, in vitro fertilization, embryo } \\
\text { selection }\end{array}$ \\
\hline
\end{tabular}

\section{SCHOLARONE" Manuscripts}




\section{Clinical Performance of a specific Granulocyte Colony Stimulating Factor ELISA to Determine its Concentration in Follicular Fluid as a Predictor of Implantation Success during In Vitro Fertilization}

H. Tournaye ${ }^{1 \mp}$, T. D’Hooghe ${ }^{2 \mp}$, G. Verheyen ${ }^{1}$, K., F. Devreker ${ }^{3}$, S. Perrier d'Hauterive ${ }^{4}$, M. Nisolle $^{4}$, J-M. Foidart ${ }^{4}$, C. Munaut ${ }^{5 \mp}$, L. Noel $^{4 \mp}$,

${ }^{1}$ Centre for Reproductive Medicine, Universitair Ziekenhuis Brussel, Vrije Universiteit Brussel, Brussels, Belgium; Laarbeeklaan 101, 1090 Brussels (Jette), Belgium

${ }^{2}$ Department of Development and Regeneration, Biomedical Sciences, Herestraat 49, Campus Gasthuisberg, KU Leuven ((University of Leuven), 3000 Leuven, Belgium. and Global Medical Affairs Fertility, Research and Development, Merck Biopharma KGaA, Frankfurter Strasse 250, 64289 Darmstadt, Germany

${ }^{3}$ Research Laboratory for Human Reproduction, Université Libre de Bruxelles, Brussels, Campus Erasme, Belgium ; Route de Lennik 808, 1070 Brussels, Belgium

${ }^{4}$ Département of Gynécologie-Obstétrique, Centre de Procréation Médicalement Assistée de l'Université de Liège, CHR Citadelle, Liège, Belgium ; B-4000 Liège, Belgium

${ }^{5}$ Laboratory of Tumor and Development Biology, GIGA-Reseach, University of Liege,B-4000 Liège, Belgium

${ }^{\top}$ The authors consider that the first and last two authors should be regarded as joint first and last authors.

\section{Corresponding author: Carine Munaut}

Postal address: Laboratory of Tumor and Development Biology, University of Liege, site SartTilman, Building 23/4, Avenue Hippocrate, 13, 4000 Liege, Belgium

E-mail: c.munaut@uliege.be

Phone: +3243662453

Fax: +3243662936 


\title{
Clinical Performance of a specific Granulocyte Colony Stimulating Factor ELISA to Determine its Concentration in Follicular Fluid as a Predictor of Implantation Success during In Vitro Fertilization
}

\begin{abstract}
This study aimed to demonstrate the clinical performance of an ultra-sensitive follicular fluid (FF) G-CSF immunoassay to confirm previous work, indicating a correlation between FF GCSF concentration and live birth potential of the corresponding embryo after in vitro fertilization.

This study was a noninterventional, prospective, diagnostic clinical multicentric study conducted between August 2012 and January 2014 with 396 single embryo transfers (SETs) from 278 subjects.

During oocyte retrieval, FF was individually collected. Embryo morphology and implantation success were evaluated.

The implantation success rate in the high G-CSF group (32.3\%) was higher than the overall rate $(27.5 \%)$. Similarly, for embryos with optimal morphology, implantation success rates were highest among those in the high G-CSF concentration category (34.5\%) compared with low (19.6\%) and intermediate (29.8\%) G-CSF concentration categories. Significant differences in mean G-CSF concentrations were observed between the study sites. To minimize bias, analyses were repeated using data from the center with the largest number of SETs. In alignment with the overall analysis, this center demonstrated a $43 \%$ greater probability of implantation for optimal embryos with high G-CSF compared to the general implantation rate among optimal embryos and a $327 \%$ increase compared with the implantation rate of optimal embryos with low G-CSF.
\end{abstract}

Keywords: granulocyte colony stimulating factor; in vitro fertilization; embryo selection 


\section{INTRODUCTION}

Despite advances in many different areas, the practice of in vitro fertilization (IVF) could be improved by increasing success rates and reducing high multiple birth rates, which contribute to prematurity and increased neonatal and childhood morbidity [1]. These issues could be minimized with single embryo transfers (SET); however, this strategy is limited by the relatively low success rate of current IVF procedures. Oocyte retrieval can be performed in about $90 \%$ of started IVF cycles and embryo transfer can be carried out in $81 \%$ of started IVF cycles, but the live birth rate/cycle is limited to only approximately $30 \%[2,3]$. This means that only about $5 \%$ of collected oocytes leading to a live birth [4].

In daily practice, clinicians and embryologists at IVF laboratories have to decide which embryos have the highest competence (i.e., the best probability to implant and to initiate a pregnancy), by primarily relying on an assessment of morphologic characteristics. Such evaluations are poorly discriminative and not predictive of subsequent birth [5]. While cell-free nucleic acids [6], micro RNAs [7], mitochondrial DNA [8], and metabolic profiling [9] are currently being investigated as methods to predict reproductive potential, current literature lacks sufficient evidence in support of concrete evaluators for embryo reproductive potential.

Recently, granulocyte colony stimulating factor (G-CSF), measured by commercially available antibody coated bead-based multiplex assay (Luminex XMap Technology from Bio-Rad), in individual FF collected during oocyte retrieval, was shown to be correlated with the birth potential of the corresponding embryo $[10,11]$. Subsequently, two retrospective studies evaluated the validity of FF G-CSF as a biomarker for oocyte and embryo selection. In both studies, the mean implantation success rate was significantly higher for embryos with higher FF G-CSF concentrations. Combining G-CSF concentration with classical morphological scoring methods resulted in a significant increase in implantation success rate [10]. While the exact 
mechanism by which FF G-CSF impacts embryo implantation success is largely unknown, several hypotheses exist. Some studies postulate FF G-CSF is involved in oocyte-uterus crosstalk that leads to the preparation of a receptive uterus [12], while others proposed that FF G-CSF may influence the mRNA content of the oocyte itself, potentially influencing immune system signalling and embryo self-repair $[13,14]$.

Thus, G-CSF quantitation, in conjunction with standard morphological assessment, appears to be an informative, non-invasive method to assess embryo competence, with potential to improve reproductive outcomes. For routine clinical application G-CSF evaluation has to be easily performed. Previous analyses with commercially available ELISA kits did not correlate with those measured by Luminex and were not predictive of embryo implantation success [15]. Thus, a more sensitive ELISA-based immunoassay has been developed. This immunoassay is based on specific proprietary antibodies that allow for a level of G-CSF quantitation that is not possible using commercially available ELISA assays.

Our study specifically assessed whether embryo implantation success rate at 12 weeks gestation (10 weeks after Day 2 or Day 3 [referred to as Day 3 from herein out] fresh SET) will increase with increasing FF G-CSF concentration, as quantified by FF-G-CSF Elisa.

\section{MATERIALS AND METHODS}

\section{Study Design}

This noninterventional, prospective clinical study was designed to evaluate the clinical performance of the FF-G-CSF immunoassay. FF were collected individually during standard IVF procedures while maintaining traceability to the corresponding oocyte. (Figure 1A).

\section{Ethical Approval}

The study protocol, including amendment, and informed consent documents, were approved by Independent Ethical Committees at each study site. The study, including informed consent 
procedures, was conducted in accordance with applicable laws and regulations, International Conference on Harmonization guidelines on Good Clinical Practices, and the ethical principles that have their origin in the Declaration of Helsinki.

\section{Patients}

Between August 2012 and January 2014, participants were recruited among women undergoing IVF procedures at 10 sites in Belgium, Denmark, and Sweden. Women aged 18 to 36 years and eligible for SET at Day 3 or Day 5 with good ovarian response to endocrine stimulation and 6 to 16 large follicles ( $\geq 14 \mathrm{~mm}$ diameter) containing oocytes and $\mathrm{FF}$ at the time of oocyte retrieval were eligible to participate. Women with a previous history of severe endometriosis and/or ovarian surgery or high follicle stimulating hormone were excluded

\section{Embryo Morphology Scoring}

After incubation, each embryo was characterized by number and size of blastomeres, fragmentation percentage, multinucleation status, cytoplasm aspect, and compaction. Based upon these data, each embryo was retrospectively scored by a central reader for efficacy assessment purposes as A (optimal), B (intermediate), C (poor), or D (discarded) as previously reported [10]. It was also documented if the embryo was frozen or freshly transferred.

\section{Follicular fluid Immunoassay}

Frozen FFs were transferred on dry-ice to the central testing laboratory (Artialis SA, Liège, Belgium), by special courier (Euro Connection Company, Alleur, Belgium). FFs were thawed and G-CSF quantification was performed in triplicate using the FF-G-CSF immunoassay. Characteristics of the assay were presented in details elsewhere [16, 17]. The results obtained with this specific ELISA assay are highly correlated with those obtained with the Luminex ${ }^{\circledR}$ technology (data not shown). 


\section{Statistical Analysis}

Statistical analysis and programming was performed using R-2.15 statistical software package, rjags-3.2 package, stats 2.15 package and JAGS-3.2 (Appendix 1). The probability of implantation success was calculated for G-CSF categories using the Bayesian beta-binomial model. Probabilities were compared across G-CSF categories using Fisher's exact test.

\section{RESULTS}

\section{Patient Disposition and Baseline Characteristics}

Of 465 women enrolled, 278 completed the study according to the protocol with 396 SETs (288 Day 3 and 33 Day 5 fresh, 64 Day 3 and 11 Day 5 frozen). The mean age \pm SD of study participants at SET was $30.9 \pm 3.37$ years. Of the 288 Day 3 fresh SETs, $72.9 \%$ were designated as morphology A, $8.7 \%$ as morphology B, and 18.4\% as morphology C. Overall, $27.4 \%$ of Day 3 embryos successfully implanted in the uterus.

\section{Clinical Performance of the FF-G-CSF Immunoassay}

The 4-parameter logistic curve generated from Day 3 fresh SETs exhibited a sigmoidal trend (Figure 1B), suggesting a correlation between G-CSF concentration and implantation success; however, the probability that the top of the curve was significantly greater than the bottom of the curve was not significant. The inflection point of the curve corresponds to $22.3 \mathrm{pg} / \mathrm{mL}$ of G-CSF, which means that above this concentration, the probability of a successful implantation is greater (mean 28.2\%) than below this concentration (mean 24.0\%). 
Using ROC curve analysis, G-CSF thresholds were selected that corresponded to the highest negative predictive value $(17.5 \mathrm{pg} / \mathrm{mL})$ and the highest positive predictive value $(48 \mathrm{pg} / \mathrm{mL})$ for implantation success. Although there was no significant difference in the probability of implantation success among G-CSF categories, the probability of embryos associated with a G-CSF concentration $>48 \mathrm{pg} / \mathrm{mL}$ was $17 \%$ greater than the overall implantation success rate (Table 1). Among embryos with morphology category A, high G-CSF concentration similarly increased the probability of implantation success by $15 \%$.

\section{Post Hoc and Exploratory Analyses}

Although not significantly different, mean G-CSF concentrations \pm SD were higher for successful than for failed implantations in every case-Day 3 fresh SETs: $42.2 \pm 21.3 \mathrm{pg} / \mathrm{mL}$ $(\mathrm{n}=79)$ versus $40.3 \pm 20.6 \mathrm{pg} / \mathrm{mL}(\mathrm{n}=209)$; Day 3 frozen SETs: $44.0 \pm 30.9 \mathrm{pg} / \mathrm{mL}(\mathrm{n}=13)$ versus 41.5 $\pm 22.3 \mathrm{pg} / \mathrm{mL}(\mathrm{n}=51)$; Day 5 SETs: $58.2 \pm 17.3 \mathrm{pg} / \mathrm{mL}(\mathrm{n}=5)$ versus $48.3 \pm 22.5 \mathrm{pg} / \mathrm{mL}(\mathrm{n}=28)$; Day 5 frozen SETs: $44.3 \pm 6.4 \mathrm{pg} / \mathrm{mL}(\mathrm{n}=4)$ versus $30.1 \pm 13.8 \mathrm{pg} / \mathrm{mL}(\mathrm{n}=7)$.

The implantation success rate curve (Figure 1C) demonstrates an approximate linear increase in implantation success rate from 25 to $75 \mathrm{pg} / \mathrm{mL}$ of G-CSF, regardless of morphology category. This linear increase is maintained to about 80 to $90 \mathrm{pg} / \mathrm{mL}$, then reaches a plateau. Correspondingly, there was a trend toward higher success rates with G-CSF $>18 \mathrm{pg} / \mathrm{mL}$ compared with $\leq 18 \mathrm{pg} / \mathrm{mL}$ ( $\mathrm{p}=0.1227$; data not shown).

\section{Clinical Performance of FF-G-CSF Immunoassay Based on Data from a Single Site}

To address statistically significant between-site differences in mean G-CSF, post hoc exploratory analyses were performed for the single site with the largest number of transfers, 114 Day 3 fresh SETs. Similar patterns were observed, although the magnitude of difference in implantation success rates among G-CSF groups was more pronounced (Figure 1D and Table 2). 


\section{DISCUSSION}

In this study, we tested the hypothesis that implantation success rates at 12 weeks gestation of Day 3 fresh SETs will increase with increasing FF G-CSF concentration, as quantified by the FF-G-CSF immunoassay. While the study failed to show statistically significant differences between all of the different G-CSF concentrations and morphological categories, these results do confirm that there is an association between the concentration of G-CSF in FF and the implantation potential of the corresponding embryo.

While this present study is an observational study, a set of 3 interventional FF-G-CSF Elisa for Embryo Selection and Fertility Improvement studies (DESTINy - NCT02593513, NCT02593461, NCT02586272) were also initiated in order to extend these preliminary findings. In particular, these studies aim to evaluate the implantation success rate, overall and within each morphological category, between interventional and control groups for elective Day 3 fresh SETs. As embryos in the interventional group are to be selected based on morphology and FF GCSF concentration (as determined by FF-G-CSF Elisa), the DESTINy studies aim to generate additional support for the correlation between FF G-CSF concentration and implantation success rate and create additional support for the use of FF-G-CSF Elisa as an adjunct to embryo morphology during IVF procedures (see $\underline{\text { ClinicalTrials.gov). }}$

Study sites were allowed to use their usual collection procedures, as long as these procedures remained internally consistent. Differences among centers, in lumen needle type, tubing length, and flushing procedures or absence of flushing introduced variability into FF dilutions. This impacted the quantification of FF G-CSF concentration and caused differences in mean G-CSF concentrations among the sites. In order to address these differences, the analyses were repeated using data from the study site with the largest number of SETs, where the same collection methods were used for all procedures. The results from this single site demonstrated a similar 
pattern to the overall data, with larger, but nonsignificant, differences in the probability of implantation success, presumably due to a reduction in protocol variability.

While variability in embryo handling procedures may attribute to the non-significance of the results, data from this non-interventional study do suggest that higher levels of FF G-CSF are associated with higher implantation success rates. If our data are confirmed in future prospective randomized trials comparing a standard morphology arm with a combined morphology/FF-GCSF arm, this FF-G-CSF immunoassay may prove to be helpful as an adjunct to morphology assessments in selecting embryos for transfer and may lead to improved pregnancy rates.

Words count: 1938 


\section{DECLARATIONS}

\section{Acknowledgements}

LN and CM are respectively MD. PhD. Student and Research Associate from the F.R.S.-FNRS (Belgium).

\section{Author's roles}

All authors contributed to the concept of this paper, analysis of results, critically reviewed each draft, and approved the final version for submission.

\section{Funding}

This study was initiated by Femalon

\section{Declarations of interest}

Financial arrangements of the authors with companies whose products may be related to the present report are listed below, as declared by the authors. H. Tournaye has received a grant from Mithra and has received personal fees for scientific advisory board membership from Finox, ObsEva, AbbfTournott, and Ferring. T. D'Hooghe is an employee of Merck and a part-time employee of University of Leuven. At the time of this study, he was a full-time employee of University of Leuven. He has received grants from Merck and Ferring and financial clinical trial support from Allergan, Roche, and Proteomika. He has been a paid consultant for WHO, Allergan, Astellas, Cartegenia, Bayer, Roche, and Proteomika. G. Verheyen, S. Perrier d'Hauterive, M. Nisolle, C. Munaut and L. Noel declare no conflicts of interest. J-M. Foidart is the Scientific Advisory Board Chair for IMCYSE and has been a consultant for Mithra and Allergan. 


\section{REFERENCES}

1. Norwitz ER, Edusa V, Park JS. Maternal physiology and complications of multiple pregnancy. Semin Perinatol. 2005 Oct;29(5):338-48. doi: 10.1053/j.semperi.2005.08.002. PubMed PMID: 16360493.

2. Sterrenburg MD, Veltman-Verhulst SM, Eijkemans MJ, et al. Clinical outcomes in relation to the daily dose of recombinant follicle-stimulating hormone for ovarian stimulation in in vitro fertilization in presumed normal responders younger than 39 years: a meta-analysis. Hum Reprod Update. 2011 Mar-Apr;17(2):184-96. doi: 10.1093/humupd/dmq041. PubMed PMID: 20843965.

3. 2012 Success Rates Graphs and Charts Slides. National Center for Chronic Disease Prevention and Health Promotion, Division of Reproductive Health; 2014. p. December 12, 2014.

4. Patrizio P, Sakkas D. From oocyte to baby: a clinical evaluation of the biological efficiency of in vitro fertilization. Fertil Steril. 2009 Apr;91(4):1061-6. doi: 10.1016/j.fertnstert.2008.01.003. PubMed PMID: 18325517.

5. Guerif F, Le Gouge A, Giraudeau B, et al. Limited value of morphological assessment at days 1 and 2 to predict blastocyst development potential: a prospective study based on 4042 embryos. Hum Reprod. 2007 Jul;22(7):1973-81. doi: 10.1093/humrep/dem100. PubMed PMID: 17496054.

6. Assou S, Gala A, Al-Edani T, et al. Non-invasive test for embryo competent selection by quantification of cell-free nucleic acids in embryo culture micro drop. Fertil Steril2014. p. e215.

7. Capalbo A, Ubaldi FM, Cimadomo D, et al. MicroRNAs in spent blastocyst culture medium are derived from trophectoderm cells and can be explored for human embryo reproductive competence assessment. Fertil Steril. 2016 Jan;105(1):225-35.e1-3. doi: 10.1016/j.fertnstert.2015.09.014. PubMed PMID: 26453979.

8. Fragouli E, Ravichandran K, Munne S, et al. Clinical implications of mitochondrial DNA quantification on pregnancy outcomes: a blinded prospective non-selection study. Presented presented at: Finland. 32nd Annual Meeting of the European Society of Human Reproduction and Embryology; Helsinki2016.

9. Tan TC, Ritter LJ, Whitty A, et al. Gray level Co-occurrence Matrices (GLCM) to assess microstructural and textural changes in pre-implantation embryos. Mol Reprod Dev. 2016 Aug;83(8):701-13. doi: 10.1002/mrd.22680. PubMed PMID: 27409576.

10. Ledee N, Gridelet V, Ravet S, et al. Impact of follicular G-CSF quantification on subsequent embryo transfer decisions: a proof of concept study [Research Support, NonU.S. Gov't]. Hum Reprod. 2013 Feb;28(2):406-13. doi: 10.1093/humrep/des354. PubMed PMID: 23223438; PubMed Central PMCID: PMCPMC3545637.

11. Ledee N, Lombroso R, Lombardelli L, et al. Cytokines and chemokines in follicular fluids and potential of the corresponding embryo: the role of granulocyte colonystimulating factor. Hum Reprod. 2008 Sep;23(9):2001-9. doi: 10.1093/humrep/den192. PubMed PMID: 18503053.

12. Herrler A, von Rango U, Beier HM. Embryo-maternal signalling: how the embryo starts talking to its mother to accomplish implantation. Reprod Biomed Online. 2003 Mar;6(2):244-56. PubMed PMID: 12676010.

13. Rutella S, Zavala F, Danese S, et al. Granulocyte colony-stimulating factor: a novel mediator of T cell tolerance. J Immunol. 2005 Dec 1;175(11):7085-91. PubMed PMID: 16301609.

14. Munne S, Velilla E, Colls P, et al. Self-correction of chromosomally abnormal embryos in culture and implications for stem cell production. Fertil Steril. 2005 Nov;84(5):132834. doi: 10.1016/j.fertnstert.2005.06.025. PubMed PMID: 16275225. 
15. Ledee N, Munaut C, Serazin V, et al. Performance evaluation of microbead and ELISA assays for follicular G-CSF: a non-invasive biomarker of oocyte developmental competence for embryo implantation [Research Support, Non-U.S. Gov't]. J Reprod Immunol. 2010 Nov;86(2):126-32. doi: 10.1016/j.jri.2010.05.003. PubMed PMID: 20594599.

16. Somers F, McHugh J. Performance verification of the Diafert ${ }^{\circledR}$ Granulocyte ColonyStimulating Factor immunoassay. Proceedings of the 24th World Congress on Controversies in Obstetrics, Gynecology \& Infertility (COGI); Nov 10-13; Amsterdam, the Netherlands2016.

17. Tournaye H, D'Hooghe T, Verheyen G, et al. Clinical performance of Diafert ${ }^{\circledR}$ to determine Granulocyte Colony Stimulating Factor concentration in follicular fluid as a predictor of implantation during In Vitro Fertilization. Proceedings of the 24th World Congress on Controversies in Obstetrics, Gynecology \& Infertility (COGI); 2016 Nov 10-13; Amsterdam, the Netherlands.2016. 


\section{Figure captions}

Figure 1. A. Study design. During standard IVF procedures (i.e., oocyte retrieval), FFs were individually collected, while maintaining traceability to the corresponding oocyte. After retrieval, FFs and oocyte(s) were separated, oocyte(s) underwent fertilization, and FFs were frozen until G-CSF quantification was performed at a central laboratory using the FF-G-CSF immunoassay. Day 2/3 embryos were selected for transfer using the standard morphological criteria at each site. Embryo transfer was conducted in the usual fashion and associated implantation success rates were recorded at 12 weeks gestation (10 weeks after SET). The results of the FF-G-CSF immunoassay were not provided to the sites or Investigators and were not used to assist in embryo selection for transfer. If the first IVF cycle under the study protocol was not successful, subjects could be included in the study for a second IVF cycle. As this was a noninterventional, diagnostic study, no safety data were collected; B - Implantation Success Rate by G-CSF Concentration for Day 3 Fresh SETs (4-Parameter Logistic Regression; all sites); C Cumulative Implantation Success Rate (All SETs).; D - Implantation Success Rate by G-CSF Concentration for Day 3 Fresh SETs (4-Parameter Logistic Regression; Single site). CRF indicates case report form; FF, follicular fluid; SET, single embryo transfer; G-CSF indicates granulocyte colony stimulating factor; CI indicates confidence interval. 
Table 1. Probability of Implantation Success by G-CSF and Morphological Categories for Day 3 Fresh SETs (ROC Curve Analysis; All Sites)

\begin{tabular}{|c|c|c|c|c|c|c|c|}
\hline \multirow{2}{*}{\multicolumn{2}{|c|}{$\begin{array}{c}\text { G-CSF Category } \\
(\mathrm{pg} / \mathrm{mL})\end{array}$}} & \multicolumn{4}{|c|}{$\begin{array}{c}\text { Successful Implantations/SETs } \\
\text { Probability of implantation Success, } \%(95 \% \mathrm{CI})^{\mathrm{a}}\end{array}$} & \multicolumn{2}{|c|}{$\begin{array}{l}\text { Increase in Probability of } \\
\text { Implantation Success }\end{array}$} \\
\hline & & $\leq 17.5$ & $17.5-48$ & $>48$ & All & $>48$ vs All & $>48$ vs $\leq 17.5$ \\
\hline \multirow{4}{*}{$\frac{\frac{2}{0}}{\frac{0}{0}}$} & All & $\begin{array}{c}4 / 27 \\
15.3 \\
(6.4-28.6)\end{array}$ & $\begin{array}{c}46 / 171 \\
27.0 \\
(21.6-32.7)\end{array}$ & $\begin{array}{c}29 / 90 \\
32.3 \\
(24.6-40.7)\end{array}$ & $\begin{array}{c}79 / 288 \\
27.5 \\
(23.3-31.9)\end{array}$ & $17 \%$ & $111 \%$ \\
\hline & $\begin{array}{c}\text { A } \\
\text { (optimal) }\end{array}$ & $\begin{array}{c}4 / 21 \\
19.6 \\
(8.3-35.7)\end{array}$ & $\begin{array}{c}37 / 125 \\
29.8 \\
(23.3-36.6)\end{array}$ & $\begin{array}{c}22 / 64 \\
34.5 \\
(25.3-44.5)\end{array}$ & $\begin{array}{c}63 / 210 \\
30.0 \\
(25.0-35.4)\end{array}$ & $15 \%$ & $76 \%$ \\
\hline & $\begin{array}{c}\text { B } \\
\text { (intermediate) }\end{array}$ & $\begin{array}{r}0 / 4 \\
\text { NA }\end{array}$ & $\begin{array}{c}3 / 13 \\
23.9 \\
(8.8-45.3)\end{array}$ & $\begin{array}{c}1 / 8 \\
14.2 \\
(2.3-39.7)\end{array}$ & $\begin{array}{c}4 / 25^{*} \\
16.5 \\
(6.9-30.6)\end{array}$ & $14 \%$ & NA \\
\hline & $\begin{array}{c}\mathrm{C} \\
\text { (poor) }\end{array}$ & $\begin{array}{r}0 / 2 \\
\text { NA }\end{array}$ & $\begin{array}{c}6 / 33 \\
18.5 \\
(9.3-31.0)\end{array}$ & $\begin{array}{c}6 / 18 \\
33.7 \\
(17.7-52.6)\end{array}$ & $\begin{array}{c}12 / 53 \\
22.8 \\
(14.4-33.1)\end{array}$ & $48 \%$ & NA \\
\hline
\end{tabular}

CI indicates confidence interval; G-CSF, granulocyte colony stimulating factor; NA, not applicable; SET, single embryo transfer

$* \mathrm{p}<0.05$ versus morphology $\mathrm{C}$

a via Bayesian beta-binomial model 
Table 2. Probability of Implantation Success by G-CSF and Morphological Categories for Day 3 SETs (Single Site)

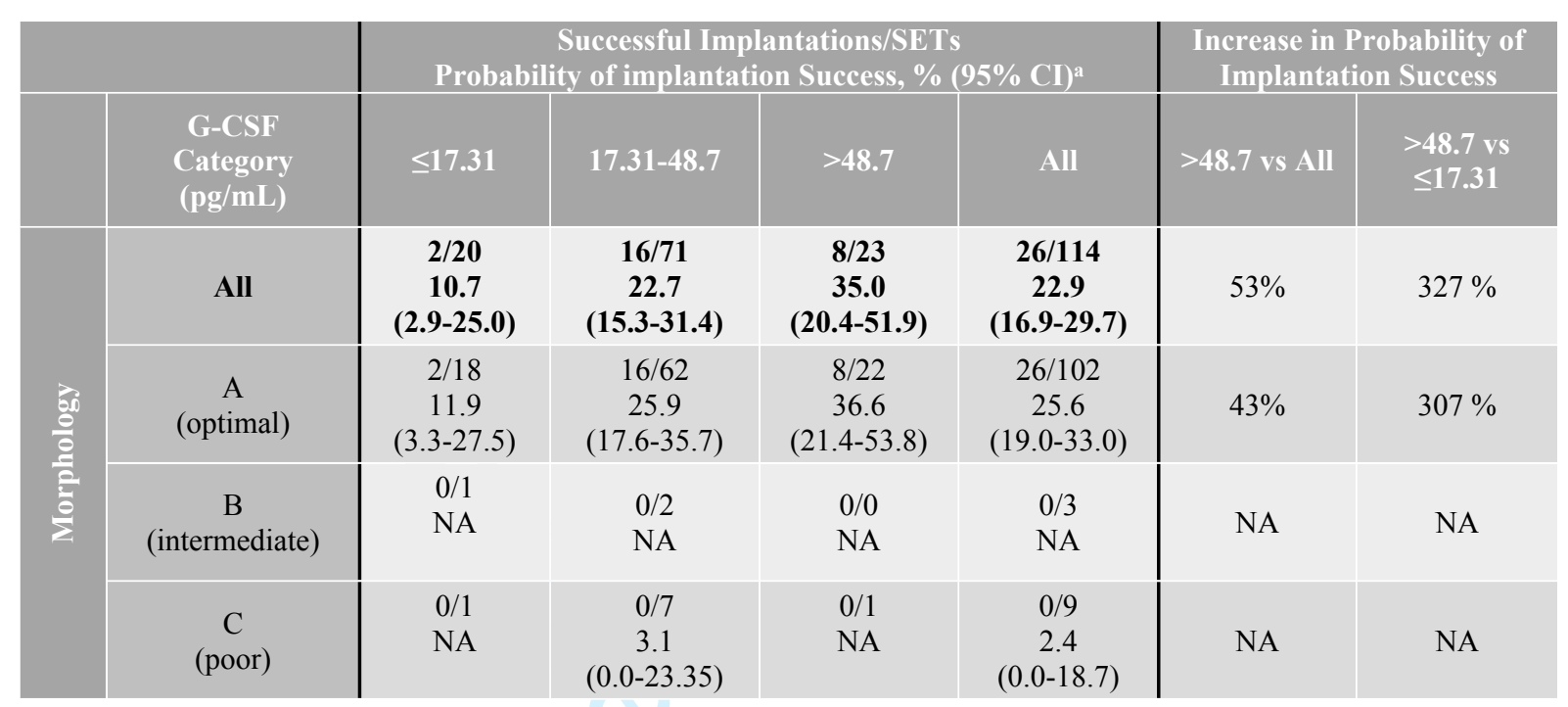

CI indicates confidence interval; G-CSF, granulocyte colony stimulating factor a via Bayesian beta-binomial model 


\section{Figure 1}

A

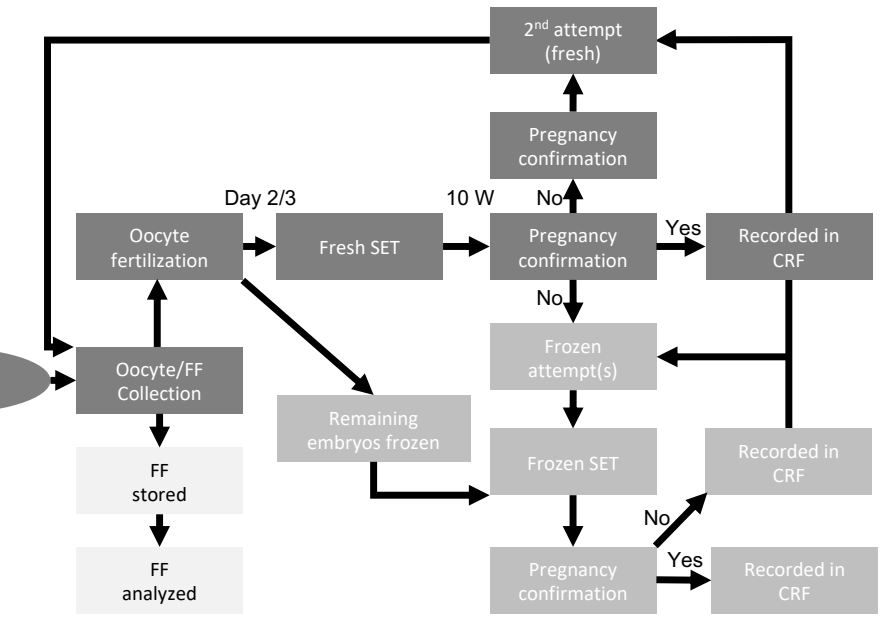

C

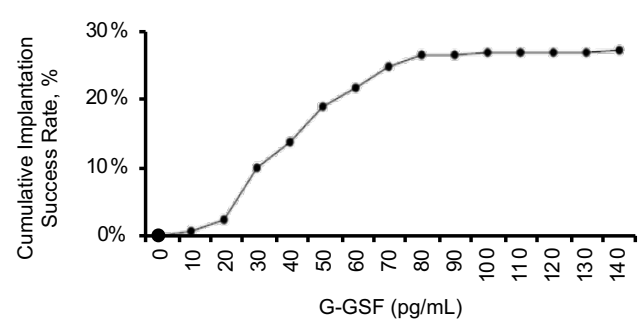

D
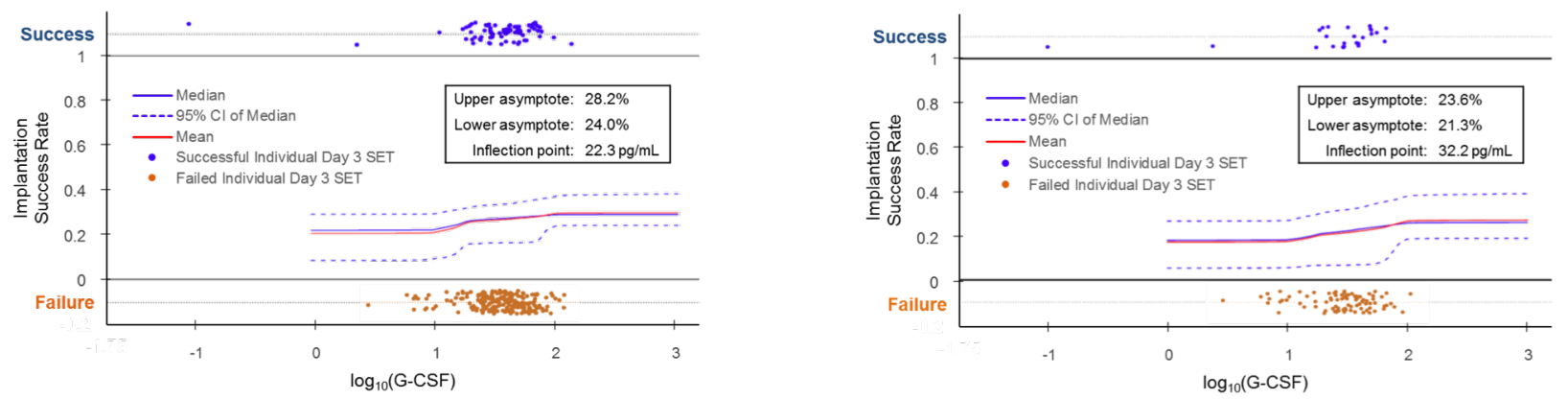


\section{APPENDIX 1}

\section{Statistical Analysis}

Sample Size

Statistical analysis and programming was provided by Arxios SPRL (Genappe, Belgium) and was performed using R-2.15 statistical software package, rjags-3.2 package, stats 2.15 package and JAGS-3.2. The estimated incidence of embryos in the different G-CSF categories was based on previous studies $[10,11]$. The sample size was evaluated by simulation of power curves based on sample sizes from 100 to 1000 (with step size of 50) and 500 to 600 (with a step size of 20). For each sample size, 20,000 virtual data sets were simulated using the proportions $0.3067,0.5357$, and 0.1582 for FF G-CSF categories and the proportions 0.3667, 0.1667 , and 0.0645 for implantation success/failure outcomes. Two one-sided Fisher exact test-based $\mathrm{p}$-values were evaluated and averaged for each virtual data set to indicates the power associated with that sample size. Based on these simulations, 300 SETs were considered sufficient for statistical separation between the bottom asymptote from the top asymptote of the 4-parameter logistic regression model with at least $80 \%$ power.

\section{Primary Statistical Analysis: 4-Parameter Logistic Regression}

The primary statistical analysis was performed using a 4-parameter logistic regression model [log (G-CSF) versus implantation success rate] with G-CSF concentration as a continuous covariate. This model was selected because, as a continuous model, it allows for differences in approximated implantation success rate for embryos falling within the same G-CSF category and because a sigmoidal model with a log scale clearly indicates a relationship between G-CSF and implantation success rate (sigmoid vs flat curve) [18]. Secondary Statistical Analysis: Receiver Operating Characteristic Curve Analysis The secondary statistical analysis was performed using a receiver operating characteristic (ROC) curve analysis to determine G-CSF thresholds based on implantation success rates 
after Day 3 fresh SETs following the method described by Lédée et al. [11]. The probability of implantation success was calculated for G-CSF categories using the Bayesian betabinomial model. Probabilities were compared across G-CSF categories using Fisher's exact test. 